\title{
Implant-related artifacts around metallic and bio-integrative screws: a CT Scan 3D Hounsfield unit assessment
}

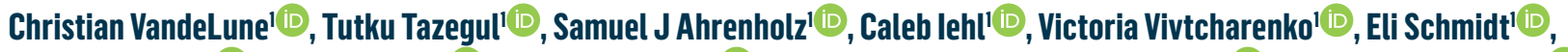

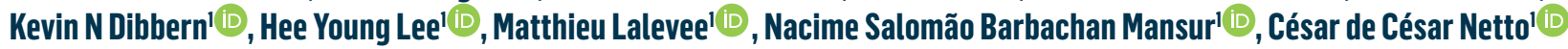 \\ 1. Department of Orthopedics and Rehabilitation, University of Iowa, Carver College of Medicine, Iowa City-IA, USA.
}

\begin{abstract}
Objective: To assess the degree of implant-related artifacts (IRA) around metallic and bio-integrative (BI) cannulated screws using Hounsfield units (HU) on computed tomography (CT). Our hypothesis was that BI implants would demonstrate significantly decreased IRA around the inserted screws.

Methods: In this cadaveric CT imaging study, we used 2 below-knee specimens. Medial displacement calcaneal osteotomy was performed, and the specimens were fixed with either metallic or BI screws. HU values were measured over 4 different lines that crossed the osteotomy position.

Results: The mean HU value was decreased in the Bl implants compared to the metallic ones in 3 different positions: near the screw, directly over the screw, and inside the screw cannula. At the line placed $1 \mathrm{~cm}$ dorsal to the screw, the HU value for the metallic screw was lower than that for the BI screw.

Conclusions: We found metallic implants to demonstrate significantly increased HU values in regions close to the implant and significantly decreased values $1 \mathrm{~cm}$ away from the implant, when compared to the BI screw. The decreased HU values $1 \mathrm{~cm}$ away from the implant could be due to a shielding effect of the surrounding bone, hindering the assessment of union and healing. BI implants represent an alternative to decrease these IRA effects.
\end{abstract}

Level of Evidence III; Case Control Study.

Keywords: Osteotomy; Calcaneus; Metals; Radiography; Tomography, x-ray computed.

\section{Introduction}

Assessment of bone healing in osteotomies, fractures, and fusions has challenged orthopedic surgeons over the years ${ }^{(1,2)}$. Accurate bone visualization is important since healing parameters are used to determine postoperative protocols and the need for surgical revision ${ }^{(3,4)}$. Normally, both clinical and radiographic findings are the basis of this evaluation, as pain, site mobility, implant failure, and bone bridging are subjectively combined to support a decision towards union or nonunion ${ }^{(5,6)}$.

Computed tomography (CT) is widely regarded as the gold-standard imaging method when appraising bone healing ${ }^{(7,8)}$. When opposing bone surfaces have contiguous trabeculation or calcific density, union is characterized in that particular area ${ }^{(7,9)}$. To estimate the rate of bone healing, the amount of bone bridging is divided by the total contact surface ${ }^{(9)}$. Although a cut-off percentage for general ossification has not yet been established, when considering arthrodesis, values above $70 \%$ are usually designated as complete unions, and values between 33 and $69 \%$ are designated as partial unions ${ }^{(6,10)}$. Bone bridging above $25-49 \%$ in hindfoot and ankle arthrodesis has been correlated to good functional outcomes ${ }^{(11)}$.

Study performed at Department of Orthopedics and Rehabilitation, University of lowa, Carver College of Medicine, lowa City-IA, USA.

Correspondence: César de César Netto. Department of Orthopaedic and Rehabilitation. University of lowa, Carver College of Medicine. 200 Hawkins Dr, John Pappajohn Pavillion (JPP). Room 01066, Lower Level. Iowa City, IA, 52242. United States. E-mail: cesar-netto@uiowa.edu. Conflicts of Interest: none Source of funding: none. Date received: July 12, 2021. Date accepted: July 14 2021. Online: August 31, 2021.

How to cite this article: VandeLune C, Tazegul T, Ahrenholz SJ, lehl C, Vivtcharenko V, Schmidt E, et al. Implant-related artifacts around metallic and biointegrative screws: a CT scan 3D Hounsfield unit assessment. J Foot Ankle. 2021;15(2):95-9. 
Implant-related artifacts (IRA) represent a significant limitation in bone and soft tissue imaging evaluations ${ }^{(12,13)}$. When assessing bone healing through conventional CT, IRA may hinder a proper and complete judgment of bridging ${ }^{(2,9)}$. Metallic implants are the standard in foot and ankle surgery but are recognized as significant sources of IRA ${ }^{(14)}$. Absorbable and bio-integrative $(\mathrm{BI})$ implants are attractive options for bone fixation when postoperative CT imaging is likely, particularly in procedures with a higher risk of nonunion. However, literature comparing the severity of IRA when using metallic or $\mathrm{Bl}$ implants is scarce. The objective of this study was to compare the degree of IRA when using metallic vs BI cannulated screws. Our hypothesis was that BI implants would demonstrate significantly decreased IRA around the inserted screws when assessed by Hounsfield units (HU) on CT.

\section{Methods \\ Design}

This cadaveric, case-control study was performed at the Orthopedic Functional Research Imaging Laboratory (OFIRL), University of lowa. Institutional Review Board approval was obtained (IRB\# 202012422).

\section{Sample}

Two fresh-frozen cadaveric lower legs obtained from knee disarticulations were used. Specimens had no deformity and were thawed for 36 hours before experimental preparation ${ }^{(15)}$.

\section{Surgical procedure}

All surgical procedures were performed by a fellowship-trained foot and ankle orthopedic surgeon with more than 10 years of experience. Using a $5 \mathrm{~cm}$ traditional oblique lateral heel approach, medial displacement calcaneal osteotomies were performed on both specimens. A cut in the safe zone of the calcaneal tuberosity was done perpendicular to the lateral wall of the calcaneus and displaced medially by $10 \mathrm{~mm}^{(16)}$. It was then provisionally fixed with two parallel Kirschner wires under fluoroscopic guidance. Positioning and placement were checked in multiple planes. The wires were used for drilling and countersinking for a set of $4.0 \mathrm{~mm}$ cannulated headless screws.

One specimen received two $4.0 \mathrm{~mm}$ standard cannulated metallic (titanium) screws (Wright Medical ${ }^{\circledR}$ ), while the other received two $4.0 \mathrm{~mm}$ cannulated $\mathrm{BI}$ fiber screws $\left(\mathrm{OSSIO}{ }^{\circledR}\right)$. The $\mathrm{BI}$ screw is composed of $50 \%$ mineral fibers (silica, magnesium, calcium, sodium oxide, boron trioxide, phosphorus) and $50 \%$ polymer (poly [l-lactide-co-d, I-lactide]) (17). A hand screwdriver was used to place both sets of screws, and their placement was continuously monitored fluoroscopically during the procedure.

\section{Cadaveric model}

Cadavers were placed in an external frame after preparation of the proximal tibia. An $80 \mathrm{lb}(356 \mathrm{~N})$ axial load was applied to the construct. Muscle forces were applied to simulate a double-legged stance with the tibia approximately perpendicular to the floor. The tension of each muscle group was set relative to the peak contractile tension of the triceps surae's strength, work percentage, and cross-sectional area. Muscle forces were then decreased proportionally to reflect axial loading to half body weight on each leg. The muscle forces applied to each tendon were: posterior tibial tendon (PTT), $40 \mathrm{~N}$; flexor digitorum longus (FDL), $22 \mathrm{~N}$; flexor hallucis longus ( $\mathrm{FHL}$ ), $22 \mathrm{~N}$; peroneus brevis and peroneus longus combined, $35 \mathrm{~N}$; and Achilles tendon, $200 \mathrm{~N}$.

\section{Weight-Bearing CT (WBCT) measurement technique}

WBCT scans were performed with a cone-beam CT lower extremity scanner (HiRise; CurveBeam, LLC, Warrington, PA, USA). Raw multiplanar de-identified data were converted into sagittal, coronal, and axial plane images and evaluated using dedicated software (CubeVue ${ }^{\mathrm{TM}}$, CurveBeam, LLC, Warrington, PA, USA). Assessments were performed by another board-certified foot and ankle surgeon with more than 10 years of experience who had experience with the dedicated software. The overall $\mathrm{HU}$ distribution within a 3D cube of $30 \mathrm{~mm}$ edge length was assessed. Additionally, within the cube, 4 linear projections were selected to sample the entire set of $\mathrm{HU}$ values along that line. Each line was parallel to the screws and crossed the osteotomy site. Line 1 was placed in close proximity to the screw (within $1 \mathrm{~cm}$ ), Line 2 was placed directly over the screws, Line 3 was placed inside the screw cannula, and Line 4 was placed dorsal to the implant by $1 \mathrm{~cm}$. The $\mathrm{HU}$ values on these lines were measured across the entire length of the line. Additionally, an $8 \mathrm{~mm}$ segment of each line as it crossed the osteotomy was selected (Figure 1).

\section{Statistical analysis}

For each measurement, data were evaluated for normality using the Shapiro-Wilk test, and descriptive statistics were obtained (mean, median, interquartile range [IQR], 95\% confidence interval values). The average $\mathrm{HU}$ value for each line was compared between metallic and BI implanted specimens by $\mathrm{t}$-tests/Wilcoxon analyses, and $\mathrm{p}$-values $\leq 0.05$ were considered significant. The JMP Statistical Software (SAS Institute ${ }^{\circledR}$ ) was used for the analysis.

\section{Results}

The specimen with metallic screws was found to have significantly higher $\mathrm{HU}$ values for lines 1,2, and 3 when compared to the $\mathrm{Bl}$ screw specimen. Values were significantly increased when considering both the entire line inside the 3D cube as well as the selected $8 \mathrm{~mm}$ line segment across the calcaneal osteotomy. When considering Line 1 (close to the screw), the metallic implant had higher $\mathrm{HU}$ values (entire, 7.26; selected, -5) when compared to $\mathrm{BI}$ (entire, -159; selected, -249). For Line 2 (over the top of the screw), the metallic implant was found to have higher $\mathrm{HU}$ values (entire, 4.84; selected, 6286) and the BI had lower values (entire, 108; selected, 151.2), as seen in figure 2. Line 3 (inside the cannula) was also found to have increased $\mathrm{HU}$ values for the metallic implant (entire, 1664; selected, 198.7) compared to the $\mathrm{BI}$ one (entire, 144; selected, -277.7). However, across Line $4(1 \mathrm{~cm}$ away from the implant), HU values were significantly decreased around the metallic implant (entire, -49: selected, -110) compared to the $\mathrm{BI}$ implant (entire, 178.5; selected, 221), as seen in figure 3. 


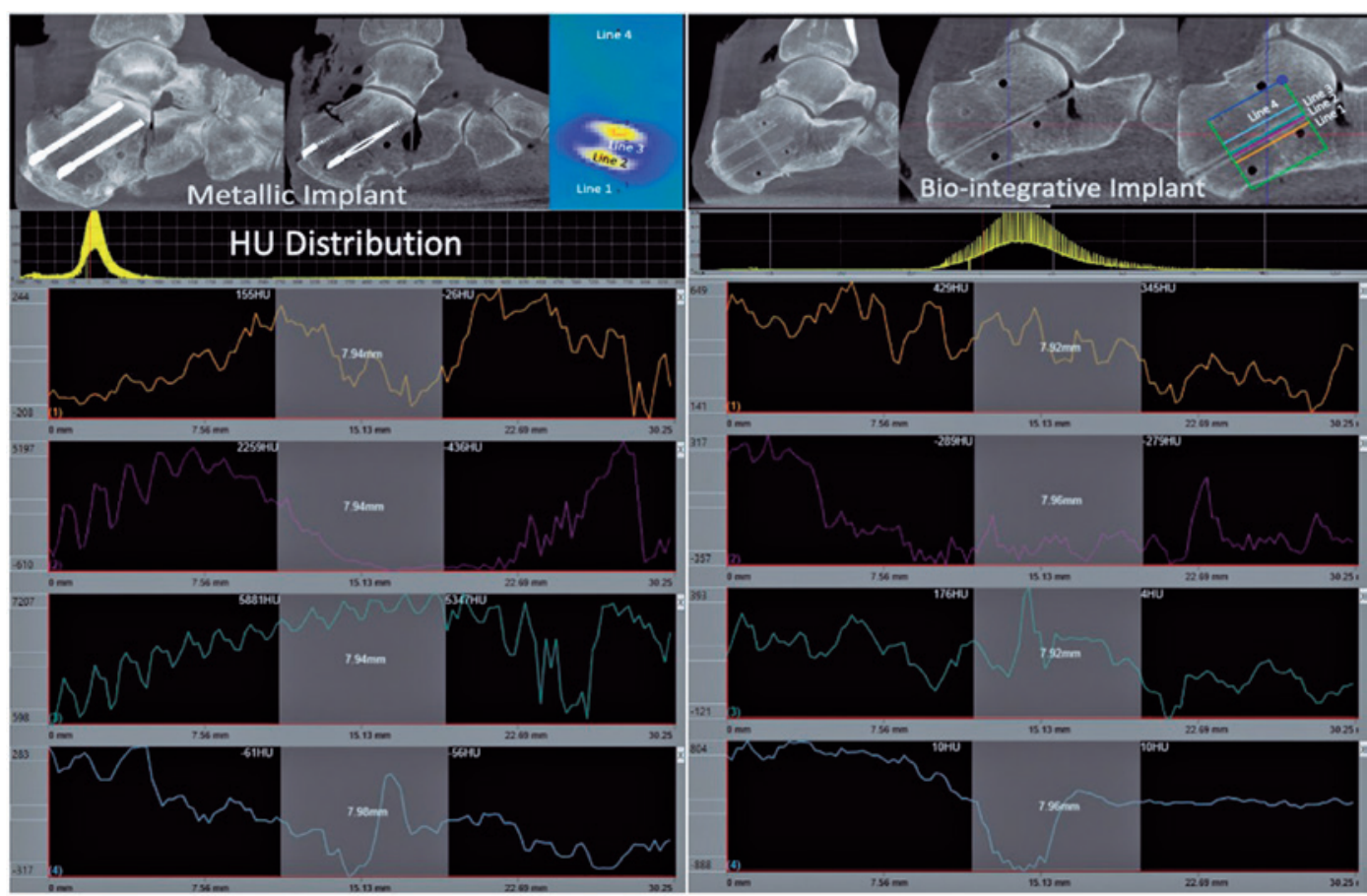

Figure 1. The left column shows data on the metallic implant and the right column, on the bio-integrative implant. Hounsfield unit distribution across Line 1 is represented by the yellow line, its distribution on Line 2 is shown by the purple line, that on Line 3, by the teal line, and that on Line 4, by the light blue line. The highlighted box $7.94 \mathrm{~mm}$ across the graph shows the "selected" line segment.
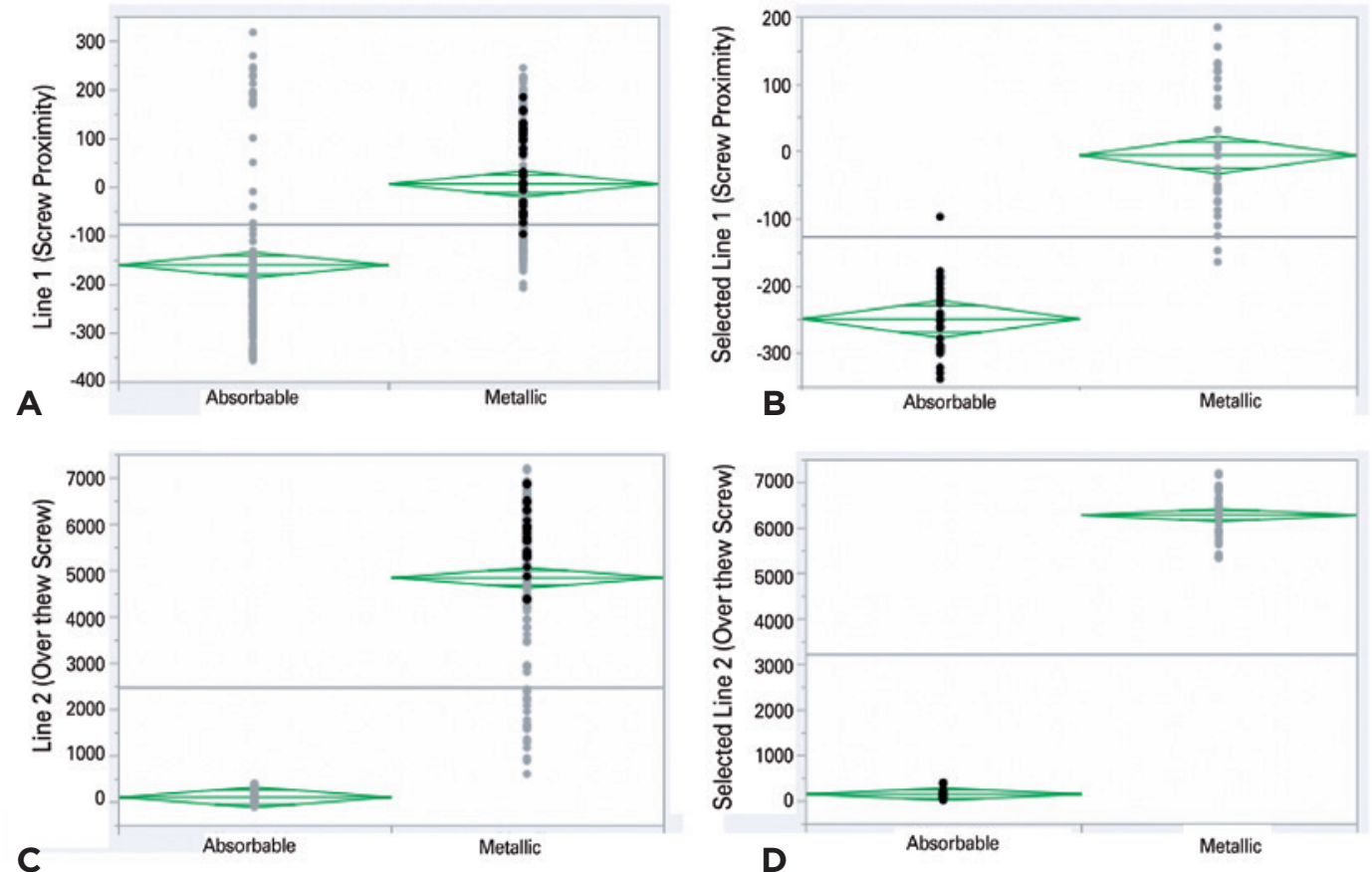

Figure 2. Line 1 Hounsfield unit values for the whole line (A) and the selected line segment (B). Line 2 values for the whole line $(C)$ and the selected line segment (D). 

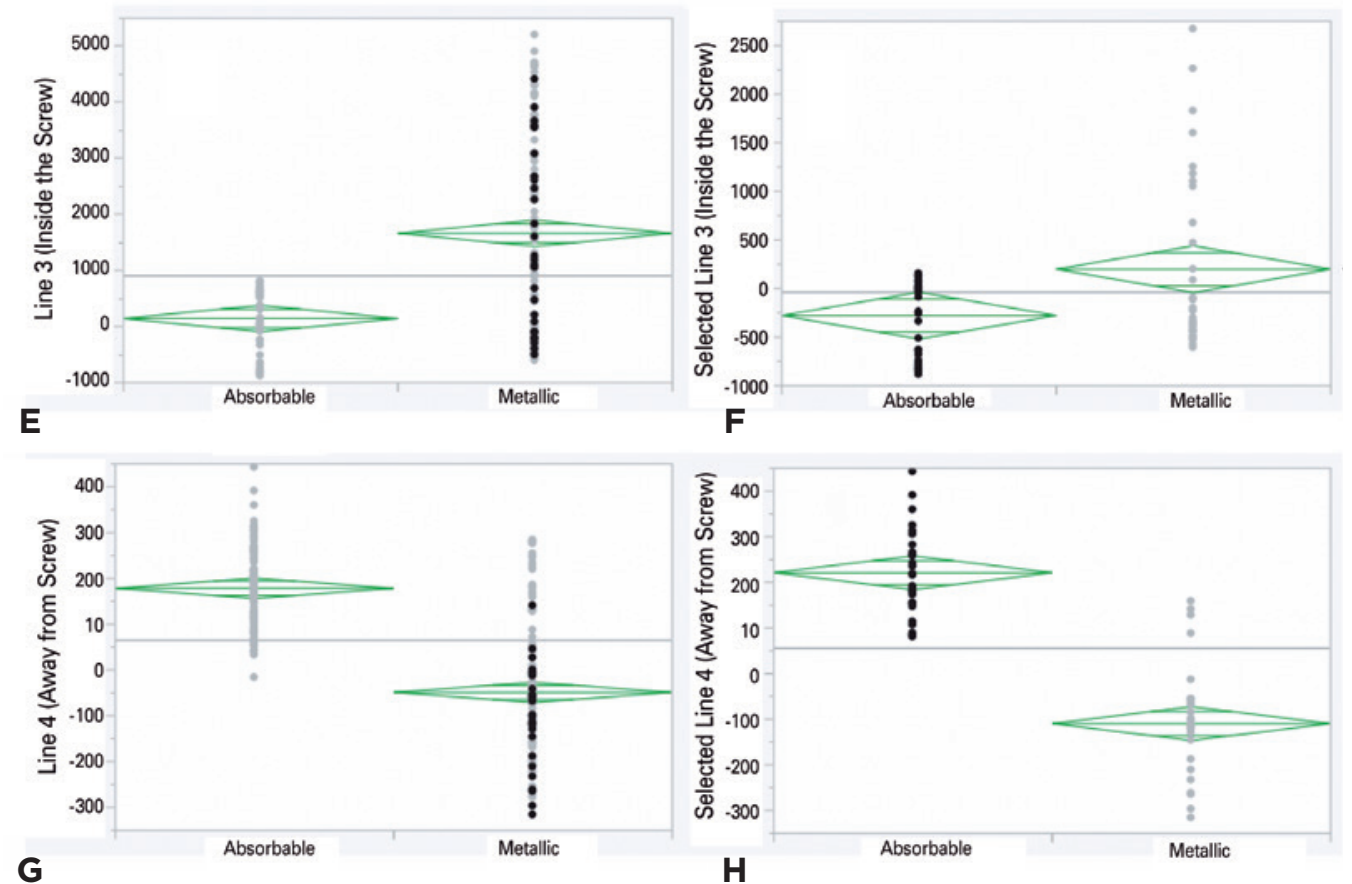

Figure 3. Line 3 Hounsfield unit values for the whole line $(E)$ and the selected line segment (F). Line 4 values for the whole line $(G)$ and the selected line segment $(H)$.

\section{Discussion}

Our cadaveric study demonstrated that BI implants significantly reduced $\mathrm{HU}$ values in 3 different planes across a calcaneal osteotomy: close to the screw, directly over the screw, and inside the screw cannula. Interestingly, $\mathrm{HU}$ values $1 \mathrm{~cm}$ away from the metallic screw (Line 4) were lower than those around the $\mathrm{BI}$ screw. This is potentially due to a beam hardening artifact, which might concentrate $\mathrm{HU}$ adjacent to the metal and shield the surrounding cancellous bone from being accurately reconstructed.

When assessing postoperative CT images for bone healing and the presence of nonunion, accurate diagnosis depends on having a clear scan close to the implant, as it crosses the osteotomy line. Historically, the lack of non-metallic implant options has led to IRA reduction through dual-energy CT scanners and post-CT imaging algorithms ${ }^{(18)}$. Metal artifact reduction software (MARS) uses algorithms that aim to reduce the effect of beam hardening and photon starvation around the implant. While these methods can reduce IRA, allowing the surrounding tissue to be better visualized, they can also add streak artifacts close to the implant and are associated with data loss ${ }^{(19,20)}$. BI implants have the possibility to remove the need for special CT protocols/software when imaging temporary hardware by reducing IRA ${ }^{(21)}$. This could also lead to better scan quality around the implant, while saving resources on imaging and processing.
Recently, other non-metallic implant options have been used surgically in the form of biodegradable, magnesium screws ${ }^{(21)}$. They have been shown to have significantly reduced IRA on postoperative CT scans and have similar efficacy to metallic implants ${ }^{(22)}$. However, when magnesium is broken down in the body, hydrogen gas is released into the surrounding tissue. The gas does not cause clinical symptoms or affect fracture healing, but can be seen radiographically(21,22). More studies are needed to compare IRA when using magnesium and BI implants.

Some clear limitations of this study are the small number of specimens used and lack of implant diversity. This was a pilot study, and sample sizing and power analyses were not performed. Procedures that require more hardware in closer proximity might show differences in IRA reduction. In addition, the procedure was done on cadaveric specimens and $\mathrm{HU}$ measurements were completed immediately after; therefore, the change in IRA once the BI screws were allowed to integrate was not tested. Furthermore, the lack of a pre-osteotomy WBCT scan makes it difficult to assess the effect of $\mathrm{BI}$ screws on IRA. For a better assessment of IRA with BI screws, a pre-osteotomy $\mathrm{HU}$ assessment should be performed. No biomechanical testing was performed either. Surgeries and readings were each performed by different professionals, but only one surgeon and one reader were involved. WBCT and the software used for the assessments are not widely available, which could affect the study's reproducibility. 


\section{Conclusions}

In this study, we compared IRA around metallic and BI implants used to stabilize medial displacement calcaneal osteotomies. We found metallic implants to demonstrate significantly increased $\mathrm{HU}$ values close to the implant and significantly decreased values $1 \mathrm{~cm}$ away from the implant. This could be due to a shielding effect on the surrounding cancellous bone. These characteristics could potentially hinder the assessment of bone density quality and bone healing. Additional clinical studies are needed to confirm and expand our findings.

Authors' contributions: Each author contributed individually and significantly to the development of this article: CV *(https://orcid.org/O000-0002-77976111) Wrote the manuscript, participated in the review process, formatted the article, made figures, approved final version; TT *(https://orcid.org/O0000002-3802-3422) Participated in the review process and formatted article; SJA *(https://orcid.org/0000-0002-5486-9858) Formatted the article, collected the data; $\mathrm{Cl}^{*}$ (https://orcid.org/0000-0003-1434-2725) Formatted the article, collected the data; VV *( https://orcid.org/0000-0002-1574-3793) Bibliographic review, data collection; ES *(https://orcid.org/O000-0002-6922-5238) Bibliographic review, data collection; HYL *(https://orcid.org/OOOO0003-4179-9501) Bibliographic review, data collection; ML *(https://orcid.org/0000-0001-5058-8867) Bibliographic review, data collection; KND *(https:// orcid.org/0000-0002-8061-4453) Participated in the review process, formatted the article; NSBM *(https://orcid.org/0000-0003-1067-727X) Participated in the review process, formatted the article, approved the final version; CCN *(https://orcid.org/0000-0001-6037-0685) Conceived and planned the activities that led to the study, participated in the review process, approved the final version, performed the surgeries. All authors read and approved the final manuscript. *ORCID (Open Researcher and Contributor ID) iD).

\section{References}

1. Yamagishi M, Yoshimura Y. The biomechanics of fracture healing. J Bone Joint Surg Am. 1955;37(5):1035-68

2. Krause F, Younger AS, Baumhauer JF, Daniels TR, Glazebrook M, Evangelista PT, et al. Clinical Outcomes of Nonunions of Hindfoot and Ankle Fusions. J Bone Joint Surg Am. 2016;98(23):2006-16.

3. Gagne OJ, Veljkovic AN, Glazebrook M, Penner M, Wing K, Younger ASE. Agonizing and Expensive: A Review of Institutional Costs of Ankle Fusion Nonunions. Orthopedics. 2020;43(4):e219-e24

4. Ziegler P, Friederichs J, Hungerer S. Fusion of the subtalar joint for post-traumatic arthrosis: a study of functional outcomes and non-unions. Int Orthop. 2017;41(7):1387-93.

5. Wilson FC Jr, Fay GF, Lamotte P, Williams JC. Triple arthrodesis. A study of the factors affecting fusion after three hundred and one procedures. J Bone Joint Surg Am. 1965;47:340-8.

6. Coulomb R, Hsayri E, Nougarede B, Marchand P, Mares O, Kouyoumdjian P, et al. Do clinical results of arthroscopic subtalar arthrodesis correlate with CT fusion ratio? Orthop Traumatol Surg Res. 2019;105(6):1125-9.

7. Coughlin MJ, Grimes JS, Traughber PD, Jones CP. Comparison of Radiographs and CT Scans in the Prospective Evaluation of the Fusion of Hindfoot Arthrodesis. Foot Ankle Int. 2006;27(10):780-7.

8. Cerrato RA, Aiyer AA, Campbell J, Jeng CL, Myerson MS. Reproducibility of Computed Tomography to Evaluate Ankle and Hindfoot Fusions. Foot Ankle Int. 2014;35(11):1176-80.

9. Dorsey ML, Liu PT, Roberts CC, Kile TA. Correlation of Arthrodesis Stability with Degree of Joint Fusion on MDCT. AJR Am J Roentgenol. 2009;192(2):496-9.

10. Jones CP, Coughlin MJ, Shurnas PS. Prospective CT scan evaluation of hindfoot nonunions treated with revision surgery and lowintensity ultrasound stimulation. Foot Ankle Int. 2006;27(4):229-35.

11. Glazebrook M, Beasley W, Daniels T, Evangelista PT, Donahue R, Younger A, et al. Establishing the Relationship Between Clinical Outcome and Extent of Osseous Bridging Between Computed Tomography Assessment in Isolated Hindfoot and Ankle Fusions. Foot Ankle Int. 2013;34(12):1612-8.

12. Kumar NM, de Cesar Netto C, Schon LC, Fritz J. Metal Artifact Reduction Magnetic Resonance Imaging Around Arthroplasty Implants: The Negative Effect of Long Echo Trains on the ImplantRelated Artifact. Invest Radiol. 2017;52(5):310-6.

13. Kosmas C, Hojjati M, Young PC, Abedi A, Gholamrezanezhad A Rajiah P. Dual-layer spectral computerized tomography for metal artifact reduction: small versus large orthopedic devices. Skeletal Radiol. 2019;48(12):1981-90

14. De Cesar Netto C, Schon LC, Da Fonseca LF, Chinanuvathana A, Stern SE, Fritz J. Metal artifact reduction MRI for total ankle replacement sagittal balance evaluation. Foot Ankle Surg. 2019; 25(6):739-47

15. Sands A, White C, Blankstein M, Zderic I, Wahl D, Ernst M, et al. Assessment of ankle and hindfoot stability and joint pressures using a human cadaveric model of a large lateral talar process excision a biomechanical study. Medicine (Baltimore). 2015;94(11):e606.

16. Talusan PG, Cata E, Tan EW, Parks BG, Guyton GP. Safe Zone for Neural Structures in Medial Displacement Calcaneal Osteotomy A Cadaveric and Radiographic Investigation. Foot Ankle Int. 2015;36(12):1493-8

17. Štalc J, Cicchinelli LD, Miller S, Richter M. Fiber-reinforced fixation implant for proximal interphalangeal joint arthrodesis shows implant bio-integration at 1-year follow-up. Foot Ankle Surg 2021:S1268-7731(21)00134-X.

18. Kasparek MF, Töpker M, Lazar M, Weber M, Kasparek M, Mang T, et al. Dual-energy CT and ceramic or titanium prostheses materia reduce $\mathrm{CT}$ artifacts and provide superior image quality of total knee arthroplasty. Knee Surg Sports Traumatol Arthrosc. 2019 27(5):1552-61.

19. Bolstad K, Flatabø S, Aadnevik D, Dalehaug I, Vetti N. Meta artifact reduction in CT, a phantom study: subjective and objective evaluation of four commercial metal artifact reduction algorithms when used on three different orthopedic metal implants. Acta Radiol. 2018;59(9):1110-18.

20. Giantsoudi D, De Man B, Verburg J, Trofimov A, Jin Y, Wang G, et al. Metal artifacts in computed tomography for radiation therapy planning: dosimetric effects and impact of metal artifact reduction. Phys Med Biol. 2017;62(8):R49-R80.

21. Sonnow L, Könneker S, Vogt PM, Wacker F, von Falck C. Biodegradable magnesium Herbert screw - image quality and artifacts with radiography, CT and MRI. BMC Med Imaging. 2017; 17(1):16.

22. Acar B, Kose O, Unal M, Turan A, Kati YA, Guler F. Comparison of magnesium versus titanium screw fixation for biplane chevron medial malleolar osteotomy in the treatment of osteochondral lesions of the talus. Eur J Orthop Surg Traumatol. 2020;30(1):163-73. 